\title{
THE APPLICATION OF LEAST SQUARE IN PRECISION CYLINDRICAL GRINDING
}

\author{
Dawei Fu, Li Su, Yang Wang \\ School of Mechanical Engineering,Hebei Unibersity of Technlolgy, China; Email: \\ ffddww@163.com.
}

\begin{abstract}
In this paper, PMAC(Programmable Multi-Axis Controller) is used as machine tool CNC system to control linear motor's micro-movement and to achieve online measurement of part circularity error. The part circularity is calculated through least square method by measured circularity error. The part circularity error can be decreased by PID control of PMAC, so precision cylindrical grinding can be achieved.
\end{abstract}

Key words: PMAC(Programmable Multi-Axis Controller); PID control ; precision grinding, LSM(Least Square Method).

\section{INTRODUCTION}

As high-speed and super high-speed grinding can greatly improve grinding efficiency and machine-part manufacturing quality, it is a kind of technology of powerful vitality and vast range of prospects. It is devoted much attention by the advanced industry countries of the world. In this paper, a LSM is proposed to fulfill high-speed precision grinding of cylindrical parts based on PMAC system. The linear motor's micro-movement and electric spindle of machine tool are controlled by open controller PMAC. Also, a set of numerical control system of grinder is integrated. A good experimental result is gotten in this grinder to grind cylindrical parts.

This project is supported by Doctor foundation of Hebei education bureau under grant B2003205.

Please use the following format when citing this chapter:

$\mathrm{Fu}$, Dawei, Su, Li, Wang, Yang, 2006, in International Federation for Information

Processing (IFIP), Volume 207, Knowledge Enterprise: Intelligent Strategies In Product Design, Manufacturing, and Management, eds. K. Wang, Kovacs G., Wozny M., Fang M., (Boston: Springer), pp. 749-754. 


\section{SYSTEM INTEGRATION OF CNC GRINDER}

As shown in Fig.1, this machine tool adopts two-axis PMAC to control linear motor and servo motor. At the same time electric spindle is cooled by circulatory water.

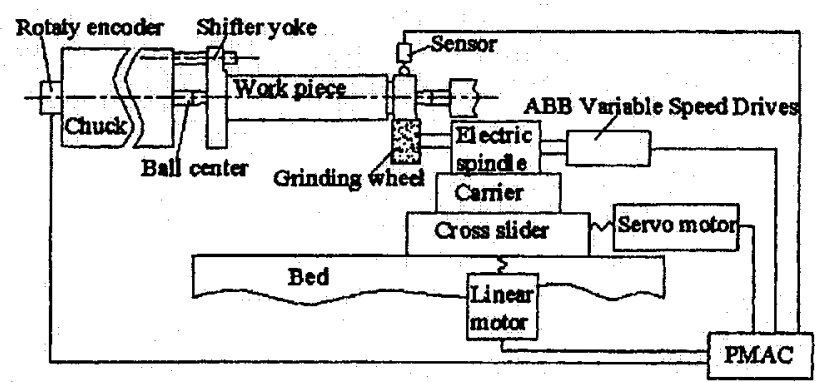

Fig.1 The principle of high-speed precislon cylindrical grinding

\section{THE MATHEMATICAL METHOD OF LEAST SQUARES CIRCLE OF CIRCULARITY ERROR}

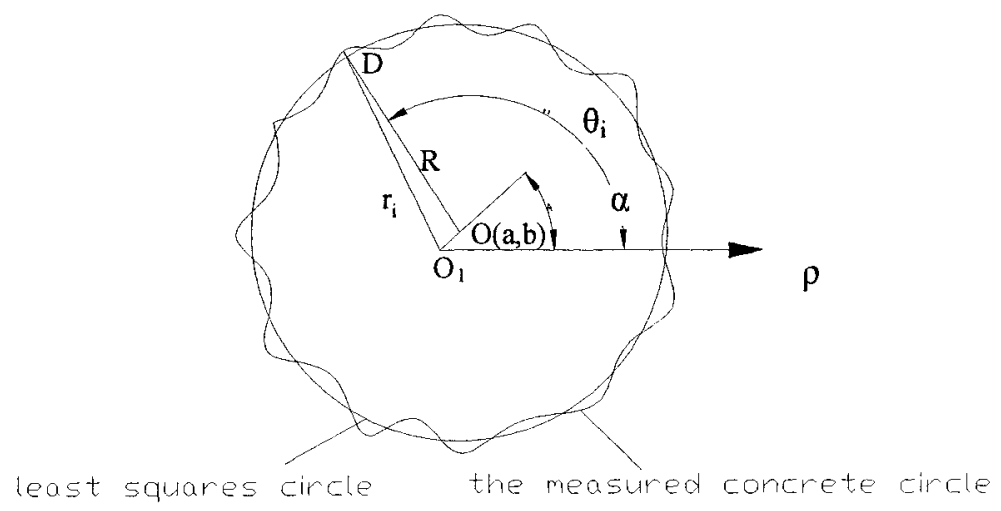

Figure2. The mathematical method of least squares circle of circle of circularity error.

As shown in fig.2, took the rotary center $O_{1}$ of the measured section as the center of a circle and divided the measured circle into $\mathrm{m}$ equal angles, radii $r_{i}(i=1,2 \ldots m)$ from the point $\mathrm{O}_{1}$ can be obtained. The points of 
intersection between these $m$ radii $r_{i}$ and the measured circle are $P_{i}$. At the pole coordinate of $\mathrm{O}_{1}$ as the origin, let the center of least squares circle as $\mathrm{O}$, the radius an $\mathrm{R}$, the offset of least squares circle and rotary center as e. The radial offset from each discrete sampling point $P i$ to least squares circle as $\epsilon_{i}$ $\left(\mathrm{i}=1,2 \mathrm{~m}\right.$ ), the angle of line $\mathrm{O}_{1} \mathrm{P}_{\mathrm{i}}$ from each equal sampling point $\mathrm{Pi}$ to rotary center $\mathrm{O}_{1}$ and the axis of pole coordinate as $\theta_{i}(\mathrm{i}=1,2 \mathrm{~m})$. The point of the intersection between line $\mathrm{OP}_{\mathrm{i}}$ and the least squares circle is $\mathrm{D}$. The radial error $\Delta \mathrm{r}_{i}$ from the center $\mathrm{O}_{1}$ is collected by the sensor. From the Fig.2, it is obtained $\mathrm{OD}=\mathrm{R}, \mathrm{OO}_{1}=\mathrm{e}, \mathrm{P}_{\mathrm{i}} \mathrm{D}=\epsilon_{\mathrm{i}}, \mathrm{O}_{1} \mathrm{P}_{\mathrm{i}}=\mathrm{r}_{\mathrm{i}}, \angle P_{i} O_{1} \rho=\theta_{\mathrm{i}}, \angle O O_{1} \rho=\alpha$, $\mathrm{a}=\mathrm{e} \cos \alpha, \mathrm{b}=\mathrm{esin} \alpha$

$$
\begin{aligned}
& \text { Also: } \\
& r_{i}=e \cos \left(\theta_{i}-\alpha\right)+\left[\left(R+\varepsilon_{i}\right)^{2}-e^{2} \sin ^{2}\left(\theta_{i}-\alpha\right)\right]^{1 / 2} \\
& =e \cos \theta_{i} \cos \alpha+e \sin \theta_{i} \sin \alpha+\left[\left(R+\varepsilon_{i}\right)^{2}-e^{2} \sin ^{2}\left(\theta_{i}-\alpha\right)\right]^{1 / 2}
\end{aligned}
$$

Make the non-linear function $\left[\left(R+\varepsilon_{i}\right)^{2}-e^{2} \sin ^{2}\left(\theta_{i}-\alpha\right)\right]^{1 / 2}$ linear.

Let $f(e)=\left[\left(R+\varepsilon_{i}\right)^{2}-e^{2} \sin ^{2}\left(\theta_{i}-\alpha\right)\right]^{1 / 2}$

By means of Maclaurin formula: $f(x)=\sum_{n=0}^{\infty} \frac{1}{n !} f^{(n)}(0) X^{n}$

$$
f(e)=R+\varepsilon_{i}-\frac{e^{2}}{2\left(R+\varepsilon_{i}\right)} \sin ^{2}\left(\theta_{i}-\alpha\right)-\frac{e^{4}}{8\left(R+\varepsilon_{i}\right)^{3}} \sin ^{4}\left(\theta_{i}-\alpha\right)
$$

If $\mathrm{e}<<\mathrm{R}$, and $\sin ^{2}\left(\theta_{i}-\alpha\right)<<1$

Therefore $f(e) \approx R+\varepsilon_{i}$ where the maximum error caused is $\frac{e^{2}}{2\left(R+\varepsilon_{i}\right)}$.

Accordingly: $r_{i}=R+a \cos \theta_{i}+b \sin a \theta_{i}+\varepsilon_{i} \quad \mathrm{i}=1,2 \quad . \mathrm{m}$ 
The structural matrix of data is:

$$
\theta=\left[\begin{array}{llll}
1 & 1 & \ldots \ldots & 1 \\
\cos \theta_{1} & \cos \theta_{2} & \ldots \ldots & \cos \theta_{m} \\
\sin \theta_{1} & \sin \theta_{2} & \ldots \ldots & \sin \theta_{m}
\end{array}\right]^{T}
$$

Coefficient matrix of normal equations is:

$$
A=\theta^{T} \theta=\theta=\left[\begin{array}{lll}
\mathrm{M} & \sum_{i} \cos \theta_{i} & \sum_{i} \sin \theta_{i} \\
& \sum_{i} \cos ^{2} \theta_{i} & \sum_{i} \cos \theta_{i} \sin \theta_{i} \\
& & \sum_{i} \sin ^{2} \theta_{i}
\end{array}\right]
$$

As $m$ is even number

Therefore $A=\operatorname{diag}[m, m / 2, m / 2]$

$A^{-1}=\operatorname{diag}[1 / m, 2 / m, 2 / m]$

Let $D=\left[r_{1}, r_{2}, \ldots \ldots, r_{m}\right]^{T}$

So constant matrix of normal equations is:

$$
B=\theta^{T} D=\left[\sum r_{i} \sum r_{i} \cos \theta_{i} \quad \sum r_{i} \sin \theta_{i}\right]^{T}
$$

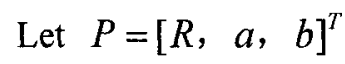

So $P=A^{-1} B=\left[\frac{1}{m} \sum r_{i}, \frac{2}{m} \sum r_{i} \cos \theta_{i}, \frac{2}{m} \sum r_{i} \sin \theta_{i},\right]^{T}$ 
That is: $R=\sum_{i} r_{i} / m=r_{0}+\Delta R$, where $\Delta R=\frac{1}{m} \sum \Delta r_{i}$

$a=\frac{2}{m} \sum_{i} r_{i} \cos \theta_{i}=\frac{2}{m} \sum \Delta r_{i} \cos \theta_{i}$

similarly: $\quad b=\frac{2}{m} \sum \Delta r_{i} \sin \theta_{i}$

So $\varepsilon_{i}=r_{i}-R-a \cos \theta_{i}-b \sin \theta_{i} \quad \mathrm{i}=1,2, \quad, \mathrm{~m}$

Or $\varepsilon_{i}=\Delta r_{i}-\Delta R-a \cos \theta_{i}-b \sin \theta_{i}$

So the circularity error of workpiece is:

$$
\begin{aligned}
& f_{o}=\max \left\{\varepsilon_{i}\right\}-\min \left\{\varepsilon_{i}\right\} \\
& =\max \left\{\Delta r_{i}-a \cos \theta_{i}-b \sin \theta_{i}\right\}-\min \left\{\Delta r_{i}-a \cos \theta_{i}-b \sin \theta_{i}\right\} \\
& \mathrm{i}=1,2, \quad, \mathrm{~m}
\end{aligned}
$$

\section{THE PID CONTROL PRINCIPLE OF PMAC}

This numerical control grinder adopts two-axis PMAC with which we can control Z-phase servomotor and X-phase linear motor's feed movement. The sensor collects workpiece circularity error signals by $A / D$ channel on the PMAC. We can attain the error on a certain intersection of workpiece. Comparing this error with tolerance of workpiece, we can figure out deviation and decide the linear motor's feed in accordance with this deviation. Finally we can attain precise machining accuracy of workpiece by many times circle count (as shown in Fig.3). Different speeds of rotation of electric-spindle are achieved by D/A channel on PMAC. The travel limits 
switch and the cooling water for electric-spindle are controlled by $\mathrm{I} / \mathrm{O}$ channel on the PMAC.

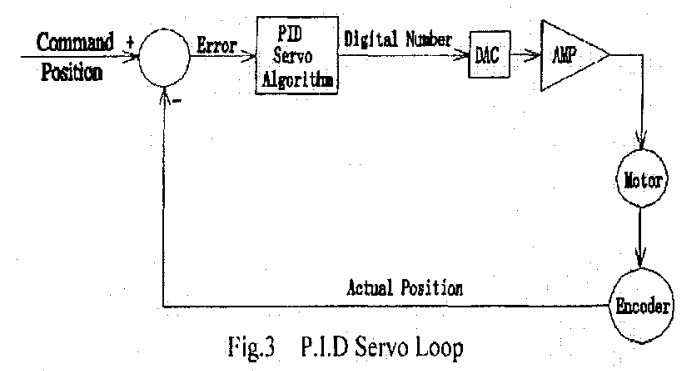

\section{CONCLUSIONS}

This paper adopts PMAC to accomplish the precision machining for grinding. The feature of PMAC is that it can control two different types of motors to accomplish coordinate motion of two axis, and it can realize some special functions such as precise linear and circle interpolator, "S-Curve" acceleration and deceleration. Thus it can fulfill high precision grinding for cylindrical, elliptical, taper contours and other kinds of irregular machine ones.

\section{REFERENCES}

1. Zhang Yu: Computer Aided Precise Measurement.(Northeastern University Press, China,1993).

2. Francis.T.Farago: Abrasive Methods Engineering. (Industrial Press, Americal, 1995).

3. Wang, YongZhang etc: Numerical Control for Machine Tool.(Harbin Institute of Technology Press, China,1995).

4. Pmac User'S Manual.(DELTA TAU Data System, Inc.1998). 